\title{
Efficacy, safety and quality of life in patients receiving subcutaneous IgG treatment: experience in Bogotá, Colombia
}

\author{
María Claudia Ortega-López*,1, Javier Garay² \& Mónica León Pinilla ${ }^{*}$ \\ ${ }^{1}$ Hospital Infantil Universitario de San José, Departamento de Pediatría, Carrera 52 \# 67A-71, Bogotá, Colombia \\ ${ }^{2}$ Pontificia Universidad Javeriana, Departamento de Epidemiología Clínica, Bogotá, Colombia \\ *Author for correspondence: Tel.: +57 320850 9623; mcol19@yahoo.com
}

\begin{abstract}
Aim: Investigate efficacy, safety and quality of life of Gammanorm ${ }^{\circledR} 16.5 \%$ (subcutaneous immunoglobulin $[\mathrm{SClG}]$ ) in patients with primary immunodeficiencies (PIDs) and safety and to lesser extent efficacy in autoimmune diseases. Patients \& methods: Medical records were extracted from 31 pediatric and 12 adult patients who received SCIG as part of the Personalized Program at University Children's Hospital, Bogotá, Colombia. Results: Mean SCIG dose was $28.7 \mathrm{~g} /$ month. Serious bacterial infections were observed in $7 / 33$ patients in the PID group, most often bacterial pneumonia (3/33). There were no serious adverse events related to SCIG treatment. Drug-related adverse reactions were reported in 2/43 patients. Conclusion: Self-administration of SCIG provided effective protection, favorable tolerability and improved quality of life in patients with PIDs and autoimmune diseases from Colombia.
\end{abstract}

First draft submitted: 26 March 2018; Accepted for publication: 1 May 2018; Published online: 15 May 2018

Keywords: primary immunodeficiencies $\bullet$ replacement therapy $\bullet$ SCIG $\bullet$ subcutaneous immunoglobulin

Genetic and clinical variability of the different primary immunodeficiencies (PIDs) includes more than 350 genetic disorders with more than 340 different gene defects [1]. These immune system alterations expose patients to recurring infections, primarily of the respiratory system [2-5].

Since the first description of PID treatment in a patient with agammaglobulinaemia [3], immunoglobulin replacement therapy has been the therapeutic standard [3-4,6]. There are different administration routes for immunoglobulin. The first one described was intramuscular, which was used for many years but abandoned due to the presence of serious local and systemic adverse drug reactions (ADRs) [5]. In the 1980s, intravenous immunoglobulin (IVIG) was adopted [7]; however, during the 1990s, the subcutaneous route (subcutaneous immunoglobulin [SCIG]) gained strength and in some countries it is now the most popular route of administration. Both routes of administration have shown an effective risk reduction of chronic and acute infections in both adults and children [8-14], as well as significant changes in health-related quality of life [15-20].

The SC route is characterized by more frequent, often weekly, but lower doses, compared with IV doses administered usually every 3-4 weeks. This results in more stable serum trough IgG levels without the large peaks seen immediately at the end of IVIG infusions. These stable IgG levels are crucial for providing an optimum protection against infections [21-25]. IVIG treatment is well tolerated by patients; however, it may be associated with systemic or local adverse reactions that affect their quality of life [26]. Likewise, in some cases, the administration can be difficult, due to complicated venous accesses, which is more common in children [8,21].

IV administration must be carried out by qualified nurses either in hospitals or health maintenance organizations, which increases costs for the healthcare system, patients and caregivers. In addition, because IV administrations last about 3-4 h, rates of school and work absenteeism are increased in this population $[21,27]$.

Clinical, cost and quality of life evaluations all support the use of SC self-administration of IgG [28-30]. Compared with IVIG, SCIG treatment is associated with reductions in the rate of injection site infections and their treatment (antibiotic therapy), as well as a decreased number of hospitalizations [31]. Similarly, studies of economic evaluations have demonstrated savings corresponding to Canadian dollars (C\$) 23.3 million in labor costs over 3 years if 
50\% of all Canadian PID patients were switched from labor-intensive hospital-based IVIG to less labor-intensive home-based SCIG therapy [30], while another Canadian study reported savings of C $\$ 5736$ per patient switched from hospital-based IVIG to home-based SCIG over 3 years [32]. The authors stated that European economic studies from France, Germany, Sweden and UK reported home-based SCIG to be 25-75\% less costly for the national healthcare systems compared with hospital-based IVIG [32].

In our local situation, pediatric services are highly aware of PIDs and their diagnosis and appropriate treatments [33] are used according to clinical guidelines [34].

This study at the University Children's Hospital of San José describes the efficacy and safety of treatment with SCIG by an infusion pump in pediatric and adult patients with PID, aligned with their quality of life, and safety and to lesser extent efficacy in adult patients with autoimmune diseases (AIDs).

\section{Methods \& management}

\section{Study design}

An observational, retrospective and descriptive study was carried out over 36 months to evaluate the efficacy, safety and quality of life in patients with PID and the safety and to lesser extent efficacy over 12 months in patients with AID receiving SCIG therapy (Gammanorm ${ }^{\circledR}$ 16.5\%).

Data were obtained from electronic clinical history records of 31 pediatric and 12 adult patients from the Personalized Program of Clinical Immunology of University Children's Hospital of San José, Colombia. These data include IgG baseline serum concentration, IgG serum concentrations during SCIG treatment, associated bacterial infections, adverse events (AEs), weekly doses of SCIG, adherence to treatment and study withdrawal with its causes. Patients attended the clinic every 3 months, with additional telephone calls or consultation visits as needed.

Each patient was empowered to care for his/her disease and apply the treatment properly. Each patient had a systematic hospital record consisting of the following information: patient's contact information, SCIG lot number, expiration date, dose (in milliliters and grams), volume to be administered and infusion site. Each patient had the contact phone numbers of people in charge of the Program, chief nurse, immunologist pediatrician and treating clinic.

\section{Patients}

Between March 2013 and March 2016, 12 adult and 31 pediatric patients with PID or AID, receiving SCIG, were included in this study (3-81 years of age).

All 31 pediatric patients received IVIG for at least 12 months prior to treatment with SCIG. IgG serum values were measured before beginning the SCIG administration (trough levels). When starting SCIG, none of the patients presented a documented viral, fungal or bacterial infection.

The inclusion criteria for changing the route of administration from IV to SC were: difficult venous access; AEs associated with IV infusion (e.g., headache, vomiting or fever); and informed consent from patients (or caregivers for patients $>7$ and $<18$ years of age) who were previously treated in the Personalized Program of Clinical Immunology of University Children's Hospital of San José.

The exclusion criteria were: any documented coagulation or hemostasis disorder, or thrombocytopenia; or patient or caregiver refused to change administration route.

\section{Treatment}

The SCIG used during the study was a $16.5 \%$ human normal immunoglobulin solution approved by the EMA, INVIMA and other regulatory authorities worldwide for home treatment (Gammanorm 16.5\%; Octapharma AB, Stockholm, Sweden).

Dosages used for PID patients varied between 100 and $200 \mathrm{mg} / \mathrm{kg} /$ week as suggested in the literature and regulatory guidelines and doses for AID varied between 200 and $400 \mathrm{mg} / \mathrm{kg} / \mathrm{week}$. All patients used the same infusion pump.

To ensure proper home treatment, patients and caregivers were trained over 6 weeks by the chief nurse in the handling of the SC infusion and possible complications at the Pediatrics Immunology Unit of the University Children's Hospital of San José. Knowledge of disease and development of dexterities were evaluated and tested to ensure proper home treatment.

Regardless of the skills shown, patients and caregivers had immediate telephone contact with the chief nurse in case questions arose during administration at home. 
Infusion pumps were individually programmed so that the required dosages for each patient were recorded in the device's memory with the maximum administration speed set to $1 \mathrm{ml} / \mathrm{min}$. Patients could not modify the programmed parameters; this could only be done by the Program's chief nurse when medically indicated.

\section{Outcome parameters}

The efficacy evaluation in PID patients was based on retrospective and observational data on IgG serum levels (concentrations before the start of SCIG treatment and during treatment) and the number of serious bacterial, viral or fungal infections (e.g., pneumonia, sepsis, meningitis and sinusitis). Further criteria were absenteeism from work and/or school and use of antibiotics at doses different to the routine prophylactic prescription. The treating physician documented the site and type of infection during the time in which the SCIG therapy was administered. In AID patients, relapses were used for efficacy assessment.

\section{Safety \& tolerability}

AEs were recorded during the time of administration and were classified as mild, moderate or severe and as local or systemic. The same classification was done for related AEs (ADRs).

According to each patient's clinical evolution, control laboratory tests were ordered: total $\operatorname{IgA}, \operatorname{IgG}, \operatorname{IgM}$ and $\mathrm{IgE}$, blood cultures and urine or cerebrospinal fluid samples, as medically indicated.

In all follow-up consultations, weight, height, blood pressure, temperature, clinical-related symptoms (if any) and any priority or emergency appointments were recorded.

Any hospitalizations were also recorded, together with the reason for hospitalization. Nevertheless, SCIG treatment was continued and administered weekly.

\section{Quality of life}

Quality of life was assessed by the Colombian version of Pediatric Quality of Life Inventory 4.0 Generic Core Scales (Peds $\mathrm{QL}^{T M}$ ) [35], a 23-item survey that assesses health-related quality of life through four domains (physical, emotional, social and school). The mean of the four scales constitutes the total summary score [36]. Validity and reliability of the PedsQL questionnaire for the patient population have been established [37,38]. The PedsQL 4.0 survey was applied by telephone only to pediatric PID patients after 1 year on SCIG.

\section{Statistical analysis}

The data presented are based on the patients' reports in the Program. They were analyzed using descriptive statistics (mean, standard deviation [SD], median, minimum and maximum). For qualitative variables, frequencies and percentages were calculated. For the analysis and presentation of quantitative measures such as trough IgG serum concentration, box plot graph types were used.

\section{Results}

We describe data from 12 adult and 31 pediatric patients (31 male, 12 female; mean age: 21.3 years; range: 3-81 years; SD: 21.8 years) treated for 36 months (PID patients) or 12 months (AID patients) with SCIG (Gammanorm 16.5\%) (Table 1). No patient stopped SCIG infusions during the 36- (PID) or 12- (AID) month observation period, respectively. In the PID group, selective antibody deficiency was the most common condition $(11 / 33)$, followed by variable immunodeficiency (8/33). In the AID group, chronic inflammatory demyelinating polyneuropathy was most frequent (4/10), followed by polymyositis (3/10). Mean SCIG dose administered was $28.7 \mathrm{~g} /$ month (range: 8-132 g/month; SD: $30 \mathrm{~g} /$ month) corresponding to $6.9 \mathrm{~g} /$ week (range: 2-33 g/week; SD: $7 \mathrm{~g} /$ week). Mean adult dose in AID was $15.21 \mathrm{~g} /$ week with an average weight of $55.8 \mathrm{~kg}(272.58 \mathrm{mg} / \mathrm{kg} / \mathrm{week})$, and pediatric dose in PID was $4.65 \mathrm{~g} /$ week $(152.45 \mathrm{mg} / \mathrm{kg} /$ week) with an average weight of $30.5 \mathrm{~kg}$.

Home administration of SCIG greatly improved patients' daily activities as school days and productive working days were not spent in emergency or unplanned medical consultations (which were reduced to nearly zero; data not shown).

The SC route of administration was effective due to fewer and/or shorter hospital stays; the time of hospitalization before and after intervention with SCIG was diminished significantly: in patients 2-4.9 years of age by $62.5 \%$, in patients $5-7.9$ years of age by $81.0 \%$, in patients $8-12.9$ years of age in $71.7 \%$ and in patients $13-18$ years of age in $88.1 \%$. On average, the time in hospital was reduced by $75.8 \%$ and also the antibiotic consumption was reduced, while a better quality of life was achieved in patients in this study. 


\section{Table 1. Demographics and baseline characteristics of treated patients.}

\begin{tabular}{|c|c|}
\hline Parameter & $n=43$ \\
\hline \multicolumn{2}{|l|}{ Gender, n (\%) } \\
\hline Female & $12(27.9)$ \\
\hline Male & $31(72.1)$ \\
\hline Mean age, years (range) & $21.3(3-81)$ \\
\hline \multicolumn{2}{|l|}{ Age groups, n (\%) } \\
\hline$<13$ years & $21(48.8)$ \\
\hline 13 to $<18$ years & $10(23.2)$ \\
\hline 18 to $<65$ years & $8(18.6)$ \\
\hline$\geq 65$ years & $4(9.3)$ \\
\hline Mean weight $(\mathrm{kg}) \pm S D$ & $38 \pm 18.7$ \\
\hline Total diagnoses $(n=43)$ & $\mathrm{N}(\%)$ \\
\hline \multicolumn{2}{|l|}{$\operatorname{PID}(n=33 ; 76.7 \%)$} \\
\hline Specific antibody deficiency & $11(33.3)$ \\
\hline CVID & $8(24.2)$ \\
\hline Hypogammaglobulinemia & $5(15.2)$ \\
\hline XLA & $4(12.1)$ \\
\hline Unspecified immunodeficiency & $1(3.03)$ \\
\hline Chronic granulomatous disease & $1(3.03)$ \\
\hline Ataxia telangiectasia & $1(3.03)$ \\
\hline DiGeorge syndrome & $1(3.03)$ \\
\hline Hyper IgM syndrome & $1(3.03)$ \\
\hline \multicolumn{2}{|l|}{$\operatorname{AID}(n=10 ; 23.3 \%)$} \\
\hline CIDP & $4(40)$ \\
\hline Polymyositis & $3(30)$ \\
\hline Myasthenia gravis & $1(10)$ \\
\hline SPS & $1(10)$ \\
\hline SID & $1(10)$ \\
\hline Mean weekly SCIG dose $(\mathrm{g} / \mathrm{kg}) \pm \mathrm{SD}$ & $6.9 \pm 7.1$ \\
\hline
\end{tabular}

AID: Autoimmune disease; CIDP: Chronic inflammatory demyelinating polyneuropathy; CVID: Common variable immunodeficiency; PID: Primary immunodeficiency; SD: Standard deviation; SID: Secondary immunodeficiency; SPS: Stiff-person syndrome; XLA: X-linked agammaglobulinemia.

\section{Table 2. Efficacy outcomes in subjects with primary immunodeficiency $(n=33)$.}

\section{End point}

Serious bacterial infections

All infection episodes

Bacterial pneumonia

Otitis media

Salmonella sepsis

Serratia tracheitis

Urosepsis
Number of subjects (\%)

7 (21.2)

$3(9.1)$
$1(3.0)$
$1(3.0)$
$1(3.0)$
$1(3.0)$

Efficacy in PID \& AID patients

Serious bacterial infections (SBIs) were observed in 7 of 33 (21\%) PID patients, with pneumonia being the most common (3/33 patients; 9\%) (Table 2). This resulted in an annualized SBI (aSBI) of approximately 0.07 , which is in line with aSBIs reported for IVIG products and well below the threshold of 1.0 set by regulatory authorities for demonstrating efficacy of immunoglobulins.

An increase in IgG plasma concentration was observed from baseline concentrations (Figure 1). The mean \pm SD serum IgG trough level at baseline was $952.2 \pm 227.3 \mathrm{mg} / \mathrm{dl}$ compared with subsequent IgG levels of 


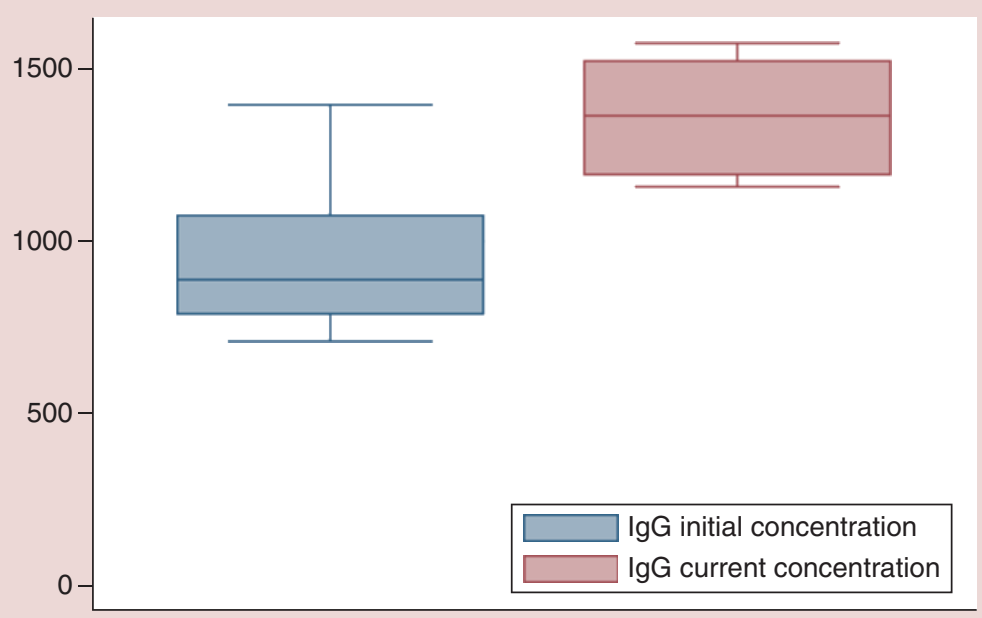

Figure 1. Median IgG trough plasma concentration $[\mathrm{mg} / \mathrm{dl}]$ of 33 primary immunodeficiency patients before and during subcutaneous immunoglobulin therapy. Boxes represent 25 and 75 percentile values. Error bars represent $95 \%$ confidence intervals.

\begin{tabular}{|l|l|}
\hline $\begin{array}{l}\text { Table 3. Adverse events observed in all subjects }(n=43) \\
\text { Type of AE }\end{array}$ & $\begin{array}{l}\text { Number }(\%) \\
\text { At least possibly related AE (ADR) }\end{array}$ \\
\hline All AEs & $2(4.7)$ \\
\hline Mild AEs & $3(39.5)$ \\
\hline Moderate AEs & $3(6.9)$ \\
\hline SAEs & $3(6.9)$ \\
\hline SAE list & $9(20.9)$ \\
\hline Bacterial pneumonia & \\
\hline Salmonella sepsis & $3(6.9)$ \\
\hline Serratia tracheitis & $1(2.3)$ \\
\hline Urosepsis & $1(2.3)$ \\
\hline Polyradiculopathy & $1(2.3)$ \\
\hline Pulmonary bullae & $1(2.3)$ \\
\hline Lupus-myelopathy & $1(2.3)$ \\
\hline ADR: Adverse drug reaction; AE: Adverse event; SAE: Serious AE. & $1(2.3)$ \\
\hline
\end{tabular}

$1259.8 \pm 400.2 \mathrm{mg} / \mathrm{dl}$. This increase was statistically significant $(\mathrm{p}<0.05)$ and together with the comparable aSBI rate demonstrates the efficacy of SCIG in treating PID patients.

All ten AID patients did not show any signs of relapse during a 12-month treatment period.

\section{Safety}

There were eight nonserious AEs (mild and moderate) considered independent of the doses of SCIG administered, and nine unrelated serious AEs: bacterial pneumonia (3), Salmonella sepsis, Serratia tracheitis, urosepsis, polyradiculopathy, pulmonary bullae and lupus-myelopathy. AEs related to infusion site reactions were mild erythema and moderate urticaria. ADRs were reported in 2 of the $43(4.7 \%)$ patients (Table 3).

\section{Patient experience: quality of life in pediatric PID patients}

In total, 22 pediatric patients (15 male, 7 female; mean age: 10.3 years; range: 4-18 years; SD: 4.6 years) completed the PedsQL 4.0 survey. Overall, patients had quality of life scores of 82.3 points with similar results in male and female patients (83 vs 81.6 points, respectively, data not shown). In girls, the domains with the lowest scores were 
Table 4. PedsQL 4 TM generic core scale: results in children with primary immunodeficiency (means [standard deviation]).

\begin{tabular}{|c|c|c|c|c|}
\hline \multirow[t]{2}{*}{ Summary score/scale } & \multirow{2}{*}{$\begin{array}{l}\text { Whole sample } \\
\text { Children with PID }(n=22)\end{array}$} & \multicolumn{3}{|c|}{ By age group } \\
\hline & & 4-7.9 years $(n=9)$ & $8-12.9$ years $(n=5)$ & $13-17.9$ years $(n=8)$ \\
\hline Total scale score (23 items) & $82.3(7.6)$ & $83.1(7.1)$ & $78.8(7.7)$ & $84(8)$ \\
\hline $\begin{array}{l}\text { Psychosocial health summary } \\
\text { score ( } 15 \text { items) }\end{array}$ & $82.5(2.5)$ & $84.1(8.4)$ & $80(3)$ & $83(2.3)$ \\
\hline $\begin{array}{l}\text { Physical health summary score } \\
\text { (8 items) }\end{array}$ & $81.7(7.6)$ & $80(7.5)$ & $76(11)$ & $86(11)$ \\
\hline $\begin{array}{l}\text { Emotional functioning scale ( } 5 \\
\text { items) }\end{array}$ & $81.9(8)$ & $90(7)$ & $69(19)$ & $85(4.1)$ \\
\hline $\begin{array}{l}\text { Social functioning scale ( } 5 \\
\text { items) }\end{array}$ & $85.2(8.1)$ & $88(11)$ & $85(13)$ & $84(8.7)$ \\
\hline $\begin{array}{l}\text { School functioning scale ( } 5 \\
\text { items) }\end{array}$ & $80.2(9.4)$ & $74.5(20)$ & $85(14)$ & $81(6)$ \\
\hline
\end{tabular}

PID: Primary immunodeficiency.

physical and school domains (70 points for both, data not shown). In boys, physical and school domains were 86 and 82 points, respectively. Most affected domain for boys was emotional functioning with 80 points.

In the analysis by age group, children in the 8-12.9 years group reported the lowest total score of 78.8 points (compared with 83.1 and 84.0 points in the other two age groups). The emotional functional scale and physical health summary score were the most affected (69 and 76 points, respectively) while in the 5-7.9 years group they were 90 and 80 points, respectively, and in the 13-17.9 years group they were 85 and 86 points, respectively (Table 4).

The pediatric patients enrolled in this study had AEs because of the disease, but after SCIG treatment they improved. These patients experienced fewer infections and hospitalizations.

\section{Discussion}

There have not been any reports in the Colombian literature about the use of SCIG in pediatric and adult patients with PID or adult patients with AID as described in the study presented here. The University Children's Hospital of San José in Bogotá is a pioneer in the registration of PID patients through the collaboration with the Latin American Society of Immunodeficiencies (LASID), and was the first to treat pediatric PID patients with SCIG.

A European SCIG - human normal immunoglobulin (Gammanorm 16.5\%) - with EMA, INVIMA and other regulatory approvals was used in this study. Its safety, efficacy and excellent tolerability has been proven for decades and was confirmed in this study in children and adults.

The patients had adequate clinical response and the safety, efficacy and tolerability seen under SCIG treatment, pediatric patients, their caregivers and adult patients also benefited with the use of SCIG and the fewer hospital admissions, interventions and comorbidities in this study.

The negative emotional implications of life-long need for IgG treatment have been reduced with SCIG compared with IVIG (data not shown). The results of Gerth and Martin [30,32] regarding economic costs for patients and their families and the healthcare system, respectively, showed a reduction between 20 and $25 \%$ for SCIG in comparison to IV administrations at the hospital. These results were an important benchmark for our study and also for future studies in our setting.

With SCIG, patients' self-care and the empowerment related to the comprehensive and timely management regarding each one of the diseases included in this study was a highly positive intangible achievement during the development of the 'Program'. The possibility of direct contact with the Program's chief and/or the treating physician marked a big difference in the attitude toward their disease. Both, patients and their caregivers felt safer and satisfied with the care of their disease, follow-up and the benefits their therapeutic adherence provided.

The benefits and efficacy of human immunoglobulin administered by both, IV and SC routes are widely discussed in literature. SCIG proved to be effective in the prevention of serious infections, and of pulmonary, neurological and systemic complications [39]. Morbidity and mortality also decreased. In general, SCIG administration decreased unplanned visits to the hospital and hospital stays due to concomitant infections [40]. 


\section{Conclusion}

Our analysis of SCIG treatment in children with PID and adults with PID and AID in Bogotá confirms the results published in the international literature: self-administered SCIG is safe, provides effective protection and favorable tolerability in pediatric PID patients and in adults with AID.

The SC route of administration was effective due to fewer and/or shorter hospital stays; the time of hospitalization before and after intervention with SCIG was diminished significantly. On average, the time in hospital was reduced by $75.8 \%$ and also the antibiotic consumption was reduced while a better quality of life was achieved in patients in this study. This evaluation is not only relevant because it is the first time the experience with SCIG for children and adults with PID and for adults with AID is published in Colombia, but also because it comprises accurate data about bacterial infections, adverse reactions and safety under SCIG treatment. All in all, SCIG is a noteworthy alternative treatment for patients with PID or AID.

\section{Summary points}

- Subcutaneous immunoglobulin (SCIG) dosages used varied between 100 and $200 \mathrm{mg} / \mathrm{kg} /$ week in primary immunodeficiency (PID) patients and 200 and $400 \mathrm{mg} / \mathrm{kg} /$ week in autoimmune disease (AID) patients and were administered by infusion pump.

- Serious bacterial infections (SBI) in PID were observed in 7/33 (21\%) PID patients, pneumonia being the most common (3/33; $9 \%)$.

- Adverse drug reactions were only reported in 2/43 (4.7\%) of PID and AID patients.

- The serum IgG trough levels significantly increased from a mean of $952 \mathrm{mg} / \mathrm{dl}$ before SCIG treatment to $1260 \mathrm{mg} / \mathrm{dl}$ after SCIG treatment $(\mathrm{p}<0.05)$.

- After SCIG treatment, pediatric PID patients had an overall quality of life score of 82.3 points (PedsQL ${ }^{T M} 4.0$ ).

- In girls, quality of life was affected most in the physical and school domains (70 points for both).

- Children in the 8-12 years group reported the lowest total PedsQL score of 78.8 points (emotional and physical domains were the most affected: 69 and 76 points, respectively).

- Of the patients with AID, $90 \%$ were treated because of neurological diseases (most common: chronic inflammatory demyelinating polyneuropathy and polymyositis) and only $10 \%$ because of secondary immunodeficiency.

\section{Author's contributions}

MC Ortega-López drafted and structured the manuscript. ML Pinilla was the nurse in charge of educating the patients and their families, application of medication and telephone monitoring of patients, and JG Fernández established the study design and was responsible for the statistical analysis. He constructed and prepared databases, based on data previously collected by the clinical team and developed and designed posters for congress presentation. He also prepared and developed the following parts of the manuscript: background, planning of research design and statistical analysis.

\section{Acknowledgements}

Thanks go to the patients who took part in this study and their families. For his assistance with the preparation of this manuscript, the authors would also like to thank S Wietek, consultant for Octapharma Pharmazeutika Produktionsges.m.b.H., Vienna, Austria, which also funded this medical writing assistance.

Financial \& competing interests disclosure

This study was funded by Fundación Alejandra Ortega López, Colombia. The authors have no other relevant affiliations or financial involvement with any organization or entity with a financial interest in or financial conflict with the subject matter or materials discussed in the manuscript apart from those disclosed.

S Wietek, Consultant Medical Affairs at Octapharma, has assisted with writing and submitting the manuscript. P Waltl, Medical Writing Services, Klagenfurt, Austria, has reviewed the manuscript as native speaker. This was funded by Octapharma, Vienna, Austria.

\section{Ethical Conduct of Research}

The authors state that they have obtained appropriate institutional review board approval of have followed the principles outlined in the Declaration of Helsinki for all human or animal experimental investigations. In addition, for investigations involving human 
subjects, informed consent has been obtained from the participants involved. No approval from an IRB/EC was needed as the data cannot be linked to a specific patient as they are completely anonymous and confidential.

\section{Open access}

This work is licensed under the Attribution-NonCommercial-NoDerivatives 4.0 Unported License. To view a copy of this license, visit http://creativecommons.org/licenses/by-nc-nd/4.0/

\section{References}

Papers of special note have been highlighted as: $\bullet$ of interest; $\bullet \bullet$ of considerable interest

1. Bousfiha A, Jeddane L, Picard C et al. The 2017 IUIS phenotypic classification for primary immunodeficiencies. J. Clin. Immunol. 38(1), 129-143 (2018).

-• The latest International Union of Immunological Societies (IUIS) publication (update of the previous one published 2 years earlier) provides a comprehensive list of more than $\mathbf{3 5 0}$ genetic causes for primary immunodeficiencies classified by now.

2. Ochs HD, Gupta S, Kiessling P, Nicolay U, Berger M. Subcutaneous IgG study group. Safety and efficacy of self-administered subcutaneous immunoglobulin in patients with primary immunodeficiency diseases. J. Clin. Immunol. 26(3), 265-273 (2006).

3. Bruton O. Agammaglobulinemia. Pediatrics 9, 722-728 (1952).

4. Stray-Pedersen A, Sorte HS, Samarakoon P et al. Primary immunodeficiency diseases: genomic approaches delineate heterogeneous Mendelian disorders. J. Allergy Clin. Immunol. 139(1), 232-245 (2017).

5. Roord JJ, van der Meer JW, Kuis W, van Furth R. Treatment of antibody deficiency syndromes with subcutaneous infusion of gamma globulin. Birth Defects Orig. Artic. Ser. 19(3), 217-221 (1983).

6. Gardulf A. Clinical experiences in primary and secondary immunodeficiencies and immune-mediated conditions using Gammanorm ${ }^{\circledR}$. Immunotherapy 8(5), 633-647 (2016).

- Provides an excellent overview on the currently available clinical data for Gammanorm ${ }^{\circledR}$ both, in patients with primary and secondary immunodeficiencies.

7. Cunningham-Rundles C, Siegal FP, Smithwick EM et al. Efficacy of intravenous immunoglobulin in primary humoral immunodeficiency disease. Ann. Intern. Med. 101(4), 435-439 (1984).

8. Chapel HM, Spickett GP, Ericson D, Engl W, Eibl MM, Bjorkander J. The comparison of the efficacy and safety of intravenous versus subcutaneous immunoglobulin replacement therapy. J. Clin. Immunol. 20(2), 94-100 (2000).

- The first systematic comparison between different IgG products being administered either intravenously or subcutaneously comparing patients in UK and Sweden.

9. Berger M. Subcutaneous administration of IgG. Immunol. Allergy Clin. North Am. 28(4), 779-802 (2008).

10. Gardulf A, Andersen V, Björkander J et al. Subcutaneous immunoglobulin replacement in patients with primary antibody deficiencies: safety and costs. Lancet 345(8946), 365-369 (1995).

11. Abrahamsen TG, Sandersen H, Bustnes A. Home therapy with subcutaneous immunoglobulin infusions in children with congenital immunodeficiencies. Pediatrics 98(6 Pt 1), 1127-1131 (1996).

12. Bhole MV, Burton J, Chapel HM. Self-infusion programmes for immunoglobulin replacement at home: feasibility, safety and efficacy. Immunol. Allergy Clin. North Am. 28(4), 821-32 (2008).

13. Leussink VI, Hartung HP, Kieseier BC, Stettner M. Subcutaneous immunoglobulins in the treatment of chronic immune-mediated neuropathies. Ther. Adv. Neurol. Disord. 9(4), 336-343 (2016).

- Describes current evidence for the clinical effectiveness and safety of subcutaneous immunoglobulin products for the off-label treatment of immune-mediated neuropathies (chronic inflammatory demyelinating polyneuropathy and multifocal motor neuropathy $[\mathrm{MMN}])$.

14. Jolles S, Borte $\mathrm{M}$ et al., Nelson RP Jr Long-term efficacy, safety, and tolerability of Hizentra ${ }^{\circledR}$ for treatment of primary immunodeficiency disease. Clin. Immunol. 250(2), 161-169 (2014).

15. Berger M, Murphy E, Riley P, Bergman GE, VIRTUE Trial Investigators. Improved quality of life, immunoglobulin G levels, and infection rates in patients with primary immunodeficiency diseases during self-treatment with subcutaneous immunoglobulin G. South. Med. J. 103(9), 856-863 (2010).

16. Nicolay U, Kiessling P, Berger $\mathrm{M}$ et al. Health-related quality of life and treatment satisfaction in North American patients with primary immunedeficiency diseases receiving subcutaneous IgG self-infusions at home. J. Clin. Immunol. 26(1), 65-72 (2006).

17. Gardulf A, Nicolay U, Math D et al. Children and adults with primary antibody deficiencies gain quality of life by subcutaneous IgG self-infusions at home. J. Allergy Clin. Immunol. 114(4), 936-942 (2004).

18. Espanol T, Prevot J, Drabwell J, Sondhi S, Olding L. Improving current immunoglobulin therapy for patients with primary immunodeficiency: quality of life and views on treatment. Patient Prefer. Adherence 8, 621-629 (2014). 
19. Gardulf A, Borte M, Ochs HD, Nicolay U, Vivaglobin Clinical Study Group. Prognostic factors for health-related quality of life in adults and children with primary antibody deficiencies receiving SCIG home therapy. Clin. Immunol. 126(1), 81-88 (2008).

20. Soresina A, Nacinovich R, Bomba $\mathrm{M}$ et al. The quality of life of children and adolescents with $\mathrm{x}$-linked agammaglobulinemia. J. Clin. Immunol. 29(4), 501-507 (2009).

21. Bezrodnik L, Gómez Raccio A, Belardinelli G et al. Comparative study of subcutaneous versus intravenous IgG replacement therapy in pediatric patients with primary immunodeficiency diseases: a multicenter study in Argentina. J. Clin. Immunol. 33(7), 1216-1222 (2013).

- The first study comparing subcutaneous immunoglobulin and intravenous immunoglobulin replacement therapy in pediatric primary immunodeficiency patients in South America.

22. Thépot S, Malphettes M, Gardeur A et al. Immunoglobulin dosage and switch from intravenous to subcutaneous immunoglobulin replacement therapy in patients with primary hypogammaglobulinemia: decreasing dosage does not alter serum IgG levels. J. Clin. Immunol. 30(4), 602-606 (2010).

23. Krivan G, Jolles $S$, Granados EL et al. New insights in the use of immunoglobulins for the management of immune deficiency (PID) patients. Am. J. Clin. Exp. Immunol. 6(5), 76-83 (2017).

24. Berger M. Incidence of infection is inversely related to steady-state (trough) serum IgG level in studies of subcutaneous IgG in PIDD. J. Clin. Immunol. 31(5), 924-926 (2011).

25. Orange JS, Belohradsky BH, Berger M et al. Evaluation of correlation between dose and clinical outcomes in subcutaneous immunoglobulin replacement therapy. Clin. Exp. Immunol. 169(2), 172-181 (2012).

26. Jolles S, Orange JS, Gardulf A et al. Current treatment options with immunoglobulin G for the individualization of care in patients with primary immunodeficiency disease. Clin. Exp. Immunol. 179, 146-160 (2015).

27. Jolles S, Borte M, Nelson RP et al. Long-term efficacy, safety, and tolerability of Hizentra ${ }^{\circledR}$ for treatment of primary immunodeficiency disease. Clin. Immunol. 150(2), 161-169 (2014).

28. Lingman-Framme J, Fasth A. Subcutaneous immunoglobulin for primary and secondary immunodeficiencies: an evidence-based review. Drugs 73(12), 1307-1319 (2013).

29. Radinsky S, Bonagura VR. Subcutaneous immunoglobulin infusion as an alternative to intravenous immunoglobulin. J. Allergy Clin. Immunol. 112(3), 630-633 (2003).

30. Gerth WC, Betschel SD, Zbrozek AS. Implications to payers of switch from hospital-based intravenous immunoglobulin to home-based subcutaneous immunoglobulin therapy in patients with primary and secondary immunodeficiencies in Canada. Allergy Asthma Clin. Immunol. 10(1), 23 (2014).

31. Hansen S, Gustafson R, Smith CIE, Gardulf A. Express subcutaneous IgG infusions: decreased time of delivery with maintained safety. Clin. Immunol. 104(3), 237-241 (2002).

32. Martin A, Lavoie L, Goetghebeur M, Schellenberg R. Economic benefits of subcutaneous rapid push versus intravenous immunoglobulin infusion therapy in adult patients with primary immune deficiency. Transfus. Med. 23(1), 55-60 (2013).

33. Ortega MC, León D, De la Hoz J. Prevalence of PID in the pediatric immunology service of San José Children's Hospital Bogotá Colombia between 2004 and 2013 - LASID Meeting 2013. J. Clin. Immunol. 33(S3), 105-139 (2013).

34. Condino-Neto BT, Costa-Carvalho AS, Grumach A et al. Series: primary immunodeficiencies in Latin America (II): guidelines for the use of human immunoglobulin therapy in patients with primary immunodeficiencies in Latin America A. Allergol. Immunopathol. (Madr.) 42(3), 245-260 (2014).

-• Provides guidelines for the use of intravenous and subcutaneous immunoglobulins for Latin America and, thus, covers also Colombia.

35. Amaya-Arias AC, Alzate JP, Eslava-Schmalbach JH. Construct and criterion validity of the PedsQL $L^{\top M} 4.0$ instrument (Pediatric Quality of Life Inventory) in Colombia. Int. J. Prev. Med. 8, 57-63 (2017).

- The authors validate the PedsQ $L^{T M} 4.0$ Instrument for its use in Colombia.

36. Varni JW, Seid M, Smith Knight T, Burwinkle T, Brown J, Szer IS. The PedsQL in pediatric rheumatology: reliability, validity, and responsiveness of the Pediatric Quality of Life Inventory Generic Core Scales and Rheumatology Module. Arthritis Rheum. 46(3), 714-725 (2002).

37. Varni JW, Seid M, Kurtin PS. PedsQL 4.0: reliability and validity of the Pediatric Quality of Life Inventory version 4.0 generic core scales in healthy and patient populations. Med. Care 39(8), 800-812 (2001).

38. Vélez CM, Villada Ramírez AC, Amaya Arias AC, Eslava-Schmalbach JH. Rasch model in the validation of the Paediatric Quality of Life Inventory TM 4.0 (PedsQL 4.0TM) in Colombian children and adolescents. Rev. Colomb. Psiquiatr. 45(3), 186-193 (2016).

39. Markvardsen LH, Harbo T. Subcutaneous immunoglobulin treatment in CIDP and MMN. Efficacy, treatment satisfaction and costs. J. Neurol. Sci. 378, 19-25 (2017).

40. Fasth A, Nyström J. Safety and efficacy of subcutaneous human immunoglobulin in children with primary immunodeficiency. Acta Paediatr. 96(10), 1474-1478 (2007). 
Short Communication Ortega-López, Garay \& Pinilla 